\title{
MiR-20a-5p promotes radio-resistance by targeting Rab27B in nasopharyngeal cancer cells
}

\author{
Dabing Huang 1,2,3, Geng Bian², Yueyin Pan ${ }^{3}$, Xinghua Han³ ${ }^{3}$, Yubei Sun ${ }^{3}$, Yong Wang ${ }^{3}$, Guodong Shen ${ }^{4}$, \\ Min Cheng ${ }^{4}$, Xiang Fang ${ }^{2}$ and Shilian $\mathrm{Hu}^{1,2,4^{*}}$
}

\begin{abstract}
Background: MicroRNAs (miRNAs) was reported to be involved in cancer radio-resistance, which remains a major obstacle for effective cancer therapy.

Methods: The differently expressed miRNAs were detected by RNA-seq experiment in nasopharyngeal cancer (NPC) cells. MiR-20a-5p was selected as our target, which was subject to finding its target gene Rab27B via bioinformatics analysis. The qRT-PCR, western blot and the luciferase reporter assays were performed to confirm Rab27B as the target of miR-20a-5p. In addition, the roles of miR-20a-5p in NPC radio-resistance were detected by transfection of either miR-20a-5p-mimic or miR-20a-5p-antagomiR. The involvement of Rab27B with NPC radio-resistance was also detected by the experiments with siRNA-mediated repression of Rab27B or over-expression of GFP-Rab27B. Wound healing and invasion assays were performed to detect the roles of both miR-20a-5p and Rab27B.
\end{abstract}

Results: MiR-20a-5p promotes NPC radio-resistance. We identified that its target gene Rab27B negatively correlates with miR-20a-5p-mediated NPC radio-resistance by systematic studies of a radio-sensitive (CNE-2) and resistant (CNE1) NPC cell lines. Repression of Rab27B by siRNA suppresses cell apoptosis and passivates CNE-2 cells, whereas overexpression of Rab27B triggered cell apoptosis and sensitizes CNE-1 cells.

Conclusions: MiR-20a-5p and its target gene Rab27B might be involved in the NPC radio-resistance. Thus the key players and regulators involved in this pathway might be the potential targets for developing effective therapeutic strategies against NPC.

Keywords: Rab27B, miR-20a-5p, Radio-resistance, Nasopharyngeal cancer

\section{Background}

The malignant tumor nasopharyngeal carcinoma (NPC) occurs in the lining of nasopharynx with a multifactorial etiology [1]. Beyond the chemotherapy [2], radiation therapy is the other major methods against cancer due to its excellent local control and increased overall survival rates [3-5]. However, owing to the high sensitivity, radiation therapy often fails in various cancers, such as NPC. The main reason is that radiation treatment can

\footnotetext{
*Correspondence: hushilianslyy@vip.126.com

${ }^{2}$ Department of Geriatrics, Anhui Provincial Hospital, Anhui Medical University, Hefei 230031, Anhui, China

Full list of author information is available at the end of the article Dabing Huang and Geng Bian are Co-first authors
}

intrinsically induce radio-resistant tumor cells, which show enhanced DNA repair ability [6]. To overcome the problem of radio-resistance, it is urgently needed to elucidate the mechanisms of radio-resistance and develop new radiosensitizers.

MicroRNAs (miRNAs) are non-coding regulatory RNAs, post-transcriptionally regulate gene expression through targeting to a panel of target genes. As the critical roles reported [7], their dysregulation is associated with human diseases, including cancer biology $[8,9]$. Notably, the emerging studies have shown that miRNAs are associated with the development of radioresistance in different type of cancers $[10,11]$, such as prostate cancer [12], esophageal cancer [13]. As one of 
the well-studied miRNAs, miR-20a has been shown to function as an oncomiR in many cancers, including lung cancer [14], hepatocellular carcinoma [15], and gastric cancer [16]. Notably, miR-20a was also found to be involved in cancer irradiation treatment [17]. For example, miR-20a was shown to induce cell radio-resistance by activating the PTEN/PI3 K/Akt signaling pathway in hepatocellular carcinoma [18].

In the present study, we performed an RNA-seq assay to detect differentially expressed genes in radio-sensitive (CNE-2) versus radio-resistant (CNE-1) NPC cell lines. We showed that miR-20a-5p promoted NPC radio-resistance via repression of Rab27B, a newly identified target of miR-20a-5p. We further performed a systematic analysis of Rab27B and miR-20a-5p for their roles in the NPC radio-resistance. The regulatory effect of miR-20a-5p on NPC cell survival and apoptosis was also detected upon irradiation.

\section{Methods \\ Cell lines}

Human nasopharyngeal cancer cell lines, CNE-1 and CNE-2 were supplied by the department of radiation oncology of Sun Yat-sen University, Guangzhou, China [19]. Cells were cultured in Dulbecco's modified Eagle's medium (DMEM) (Gibco, USA) supplemented with 10\% fetal bovine serum (Gibco, USA) in a humid atmosphere containing $5 \% \mathrm{CO}_{2}$ at $37^{\circ} \mathrm{C}$.

\section{RNA-Seq analysis}

RNA-seq analysis was performed by BGI-Tech (Shenzhen, China). RNA was purified and fragmented to construct the RNA-seq library for sequencing. The sense and anti-sense cDNA molecules were synthesized. After agarose gel electrophoresis, suitable fragments were used as templates for PCR amplification. Real-Time PCR System was used in quantification and qualification of the sample library. Finally, the library was subjected to sequencing using Illumina HiSeq 2000 (Illumina, USA). The singleend library was prepared following the protocol of the IlluminaTruSeq RNA Sample Preparation Kit (Illumina) [20].

\section{Cell reagents}

The Homo sapien miR-20a-5p mimics, miR-20a-5p antagomiRs and miR-20a-5p scrambled nega-tive control (NC) were obtained from Guangzhou Ribobio, China. All the transfection experiments were performed using the Lipofectamine 2000 transfection reagent (Invitrogen Life Technologies), which was described previously [21]. Western blot and qRT-PCR assays were performed to confirm the effect of Rab27Bon the expression of miR20a-5p. The sequences used in this study are as follows:
si-Rab27B

5' - CAGUAGGAAUAGACUUUCG dTdT-3'

$3^{\prime}$-dTdT GUCAUCCUUAUCUGAAAGC-5';

hsa-miR-20a-5p

antagomiR:5'-CUACCUGCACUAUAAGCACUUUA-3'

mimic:

sense 5'-UAAAGUGCUUAUAGUGCAGGUAG-3'

antisense 5'-CUACCUGCACUAUAAGCACUUUA- ${ }^{\prime}$

\section{Irradiation and clonogenic assay}

Cells treated with miRNAs were seeded on 6-well plates in triplicate and exposed to radiation at the doses indicated using a 6-MV x-ray generated by a linear accelerator (Varian trilogy at a dose rate of $200 \mathrm{cGy} / \mathrm{min}$ ). After incubation at $37^{\circ} \mathrm{C}$ for 14 days, cells were fixed in $100 \%$ methanol and stained with $0.1 \%$ crystal violet. Colonies containing $>50$ cells were counted under a light microscope. The surviving fraction was calculated as described previously $[13,18]$. At least three independent experiments were performed to calculate the means and standard deviations.

\section{RNA analysis}

Total RNA was extracted using Trizol (Vazyme). For the mRNA analysis, the cDNA primed by oligo-dT was made with RT reagent kit (Tiangen, China), and the mRNA level of Rab27B was quantified by a duplex-qRT-PCR analysis where the TaqMan probes with a different fluorescence for $\beta$-actin (Shing Gene, China) were used in the FTC-3000P PCR instrument (Funglyn, Canada). The miRNA expression level was normalized using U6 small nuclear RNA (HmiRQP9001) as an internal control, as previ-ously described [22]. Using the $2^{-\Delta \Delta \mathrm{Ct}}$ method, the $\beta$-actin level was normalized before comparing the relative level of the target genes. The sequences of primers and probes used for the qRT-PCR analysis are as follows:

hRab27BF, 5'-GGGACACTGCGGGACAAG-3';

hRab27BR, 5'-CAGTTGGCTCATCCAGTTTCTG-3';

hRab27B probe, $5^{\prime}$-ROX-CGGTTCCGGAGTCTCACC ACTGC-3';

hACTB F: 5'-GCCCATCTACGAGGGGTATG-3'

hACTB R: 5'-GAGGTAGTCAGTCAGGTCCCG-3'

hACTB probe: 5'-CY5-CCCCCATGCCATCCTG CGTC-3'

\section{Western blotting assays}

Total proteins were extracted from cultured cells with cell lysis buffer (60 mM Tris-HCl, pH 6.8, 2\% SDS, 20\% glycerol, $0.25 \%$ bromophenol blue, and 1.25\% 2-mercaptoethanol) and heated at $95{ }^{\circ} \mathrm{C}$ for $10 \mathrm{~min}$. The heated proteins were separated by $10 \%$ SDS-PAGE gel and transferred to polyvinylidene difluoride (PVDF) membranes. After blocking with 5\% non-fat milk in TBST for $2 \mathrm{~h}$, 
the membranes were incubated overnight at $4{ }^{\circ} \mathrm{C}$ with diluted Anti-Rab27B primary antibody (13412-1-AP; SanYing, China). Followed by washing with TBST buffer three times, the membranes were incubated with secondary antibody (SA00001-2; SanYing, China) at $37^{\circ} \mathrm{C}$ while shaking on a rotary for $2 \mathrm{~h}$. The relative density (level) of proteins over the GAPDH (10494-1-AP; SanYing, China) band was quantified with the Gel-Pro Analyzer (Media Cybernetics).

\section{Cell apoptosis analysis}

Apoptosis was analyzed using Annexin V/PI double staining. After transfection for $48 \mathrm{~h}$, the cells in the logarithmic growth phase were harvested and rinsed twice with ice-bathed PBS, then FITC-labeled enhanced annexin $\mathrm{V}(3 \mu \mathrm{l})$ and propidium iodide $(3 \mu \mathrm{l})$ were added to the cell suspension at the final volume of $150 \mu \mathrm{l}$. After incubation for $30 \mathrm{~min}$, flow cytometry was performed on a FACS Calibur instrument. The number of apoptotic and necrotic cells were calculated by flow cytometry (Becton-Dickinson, USA) and analyzed by Flowjo Software. The ratio of early apoptosis was used for the test results. The experiments were performed three times independently, and a representative is shown.

\section{Luciferase reporter assay}

Cells were seeded in 24-well plate at a concentration of $2 \times 10^{5}$ cells/per well and co-transfected $24 \mathrm{~h}$ later with pGL3-luc-Rab27B UTR WTand miR-20a-5pmimic/ antagomir or NC. After transfection for $48 \mathrm{~h}$, cells were collected, and the relative luciferase activity was performed using Dula-Luciferase Reporter Assay Kit (Promega). The relative firefly luciferase activities of the UTR construct was analyzed as previously reported [23].

\section{Wound-healing assays}

For cell motility assays, cells stably expressing mimics, antagomiRs or NC were seeded in 24-well plates and cultured to near confluence. After culture for $6 \mathrm{~h}$ in DMEM without FBS, a linear wound was carefully made using a sterile $10 \mu \mathrm{l}$ pipette tip across the confluent cell monolayer, and the cell debris was removed by washing with phosphate-buffered saline. The cells were incubated in DMEM plus $10 \% \mathrm{FBS}$, and the wounded monolayers were then photographed at $0,8,24$ and $48 \mathrm{~h}$ after wounding.

\section{Invasion assays}

According to the manufacture's description, cell invasion assays were performed in a 24-well Transwell Chambers with $8 \mathrm{~mm}$ pore size chamber inserts (Corning, USA). In the assay, $1 \times 10^{4}$ cells were seeded into the upper chamber with $200 \mu \mathrm{l}$ of DMEM without FBS. In the lower chamber, $600 \mu \mathrm{l}$ of DMEM supplemented with $10 \%$ FBS was added. After incubation for $40 \mathrm{~h}$ at $37{ }^{\circ} \mathrm{C}$ and $5 \%$ $\mathrm{CO}_{2}$, the non-invading cells were removed from the plate with cotton stick.The cells that moved to the bottom surface were stained with $0.1 \%$ crystal violet for $30 \mathrm{~min}$ at $37^{\circ} \mathrm{C}$. The cells were then imaged and counted in at least 5 random fields using a CKX41 inverted microscope (Olympus, Tokyo, Japan). The assays were conducted three independent times.

\section{Immunofluorescent staining for $\mathrm{\gamma}-\mathrm{H} 2 \mathrm{AX}$}

Twenty-four hours following transfection with miR20a-5p mimic or miRNA mimic negative control, $1 \times 10^{5}$ cells were seeded in chamber slides and incubated overnight. The cells were subsequently exposed to 4 Gy irradiation (IR). Twenty-four hours following IR, the cells were fixed in $4 \%$ paraformaldehyde, permeabilized in $0.1 \%$ Triton X-100 (Sigma), blocked in $2 \%$ bovine serum albumin and incubated with a primary antibody against $\gamma$-H2AX (SanYing, China) overnight at $4{ }^{\circ} \mathrm{C}$. The primary antibody was subsequently washed off, and a secondary antibody conjugated to fluorescein isothiocyanate was applied to the slides. Cells were washed with phosphate-buffered saline and counterstained with DAPI. The $\gamma$-H2AX foci were observed under a fluorescence microscope (Olympus). For each group, the $\gamma-\mathrm{H} 2 \mathrm{AX}$ foci were counted in $\geq 50$ cells.

\section{Statistical analyses}

The data are presented as the mean, and the error bars indicate the SD. All statistical analyses were performed with Excel (Microsoft, Redmond, WA, USA). Two-tailed Student's $t$ test, a one-way analysis of variance or MannWhitney $U$ test was used to calculate statistical significance. A $P$-value of $<0.05$ was considered significant.

\section{Results}

Rab27B negatively regulates the NPC radio-resistance

We select two NPC cell lines, CNE-1 and CNE-2 as our targets, which were reported as relatively radio-resistant and radio-sensitive cell lines of nasopharyngeal cancer (NPC), respectively [19, 24-26]. We first detected the radio-sensitivity of these two cell lines and found that CNE-1 cells are more radio-resistant than CNE-2 cells (Fig. 1a). To detect the differentially expressed miRNAs in NPC cells, we performed an RNA-seq assay of CNE-1 and CNE-2 cells (Additional file 1: Table S1). The level of miR-20a-5p was over fourfold higher in CNE-1 cells than that in the CNE-2 cells. We thus further tested the expression of miR-20a-5p by qRT-PCR, which gave a 3.52-fold higher expression in CNE-1 cells (Fig. 1b). Afterwards, we predicted the target genes of miR-20a-5p based on the literature and websites. The predicted target mRNAs were further subject to comparing the expression pattern 


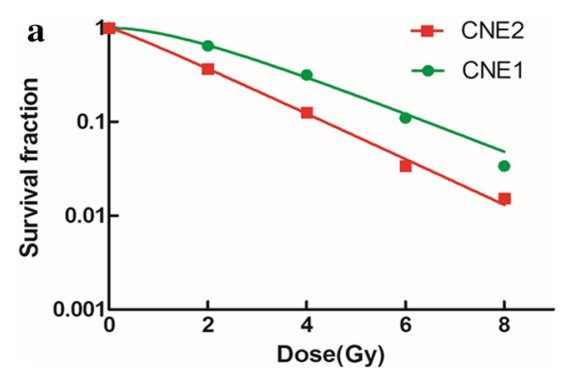

\begin{tabular}{cccc}
\hline bene & cell lines & miR-omic & qRT-PCR \\
\hline \multirow{2}{*}{ miR-193a-3p } & CNE-1 & 4.26 & $3.52 \pm 0.714$ \\
& CNE-2 & 1.00 & $1.00 \pm 0.412$ \\
\hline
\end{tabular}

\begin{tabular}{ccc}
\hline Rab27B & CNE-1 & CNE-2 \\
\hline RNA-seq & 1.00 & 1.34 \\
qRT-PCR & $1.00 \pm 1.074$ & $4.93 \pm 0.775$ \\
Western & 1.00 & 1.45 \\
\hline
\end{tabular}

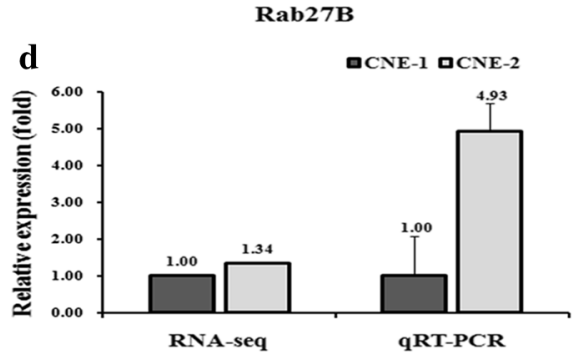

e

\section{CNE-1 CNE-2}

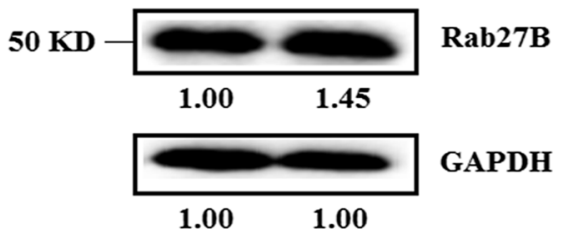

Fig. 1 Different expression patterns of miR-20a-5p/Rab27B in nasopharyngealcells CNE-1 and CNE-2. a The survival fraction of CNE-1 and CNE-2 NPC cells treated as described. The surviving fraction was calculated using the multitarget single-hit model: $Y=1-\left(1-\exp \left(-k^{*} x\right)\right) \wedge N$. The miR20a-5p expression levels in CNE-1 and CNE-2 cells were analyzed by miR-seq and qRT-PCR analyses in table (b). The expression level of Rab27B is higher in CNE-2 cells than in CNE-1 cells, as summarized in table (c). qRT-PCRandWestern blot analyses are shown in plots $\mathbf{d}$ and $\mathbf{e}$, respectively

between CNE-1 and CNE-2 cells by RNA-seq analysis. Dozens of common genes have been found which show a drastically different expression pattern in the two cell lines. Among them, the Rab27B gene is one of the significantly differentially expressed genes that negatively correlate with the expression of miR-20a-5p. Consequently, the expression level of Rab27B was higher in CNE-2 than CNE-1 at both mRNA (RNA-seq based miR-omic: 1.34:1, and qRT-PCR analysis: 4.93:1) and protein level (western blot: 1.45:1) (Fig. 1c-e). The lower expression of Rab27B in radio-resistant CNE-1 cells suggests that Rab27B is a negative regulator of NPC radio-resistance.

\section{The Rab27B gene is a target of miR-20a-5p in NPC cells}

We found that Rab27B negatively correlates with the level of miR-20a-5p, which indicated that miR-20a-5p might regulate the expression of Rab27B. To check whether Rab27B is one of the authentic targets of miR-20a-5p, we determined the Rab27B level in the miR-20a-5p mimic transfected CNE-2 and the antagomiR transfected CNE-1 cells versus the NC (scramble sequence control) transfected. The transfection of miR-20a-5p mimic in CNE-2 cells increased its expression to over fourfold (Fig. 2a), whereas the transfection of miR-20a-5p
antagomiR in CNE-1 cells significantly decreased its level to $81 \%$ (Fig. 2a). Following the changes of the miR-20a-5p level, a miR-20a-5p mimic transfection brought down the Rab27B mRNA to $80 \%$ (Fig. $2 \mathrm{~b}$ ) and protein to nearly $16 \%$ (Fig. 2c) compared to that in the NC transfected CNE-2 cells. As expected, the transfection of miR-20a-5p antagomiR increased the mRNA level of Rab27B by 5.36 fold (Fig. 2b) and the protein level by 1.85 fold in CNE-1cells (Fig. 2c).

Sequence analysis revealed that $3^{\prime}$-UTR region of Rab27B contains one potential binding motif (from 627 to $649 \mathrm{bp}$ ) for miR-20a-5p (Fig. 2d). To further conclude whether Rab27B is a direct target of miR-20a-5p, we put the wild-type Rab27B gene at the downstream of the Renilla luciferase gene of pGL3-control vector (Promega) to create pGL3-Rab27B UTR WT (Fig. 2d). These constructs were transfected into CNE-2 and CNE-1 cells, respectively, to compare the luciferase activity. The pGL3-Rab27B-UTR WT gave the relative luciferase activity of 0.78 (Fig. 2e). The transfection of miR-20a-5pmimic into CNE-2 cells significantly brought down the luciferase activity of pGL3-Rab27B-UTR WT construct, whereas the control cells showed almost the same activity upon the transfection of miR-20a-5p-mimic (Fig. 2e). 


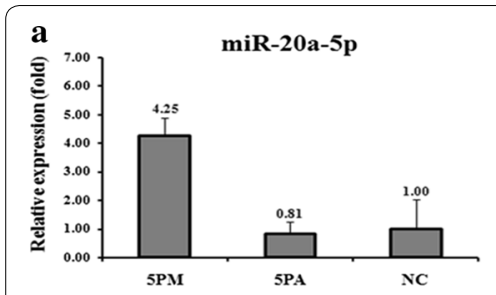

c

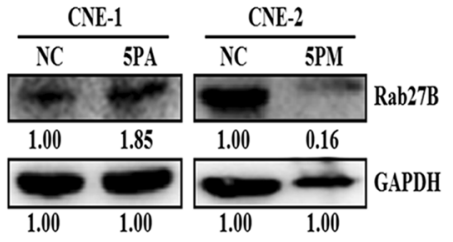

b
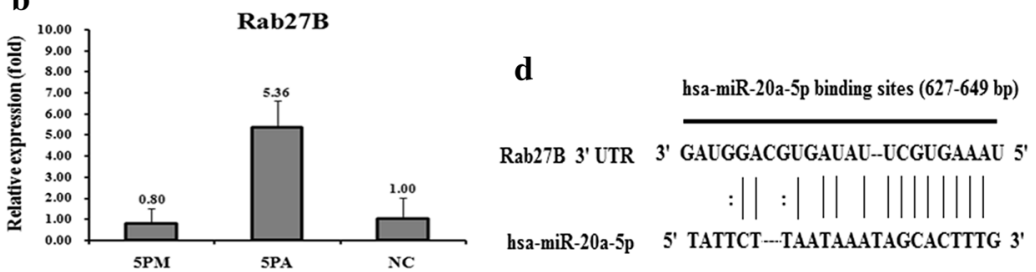

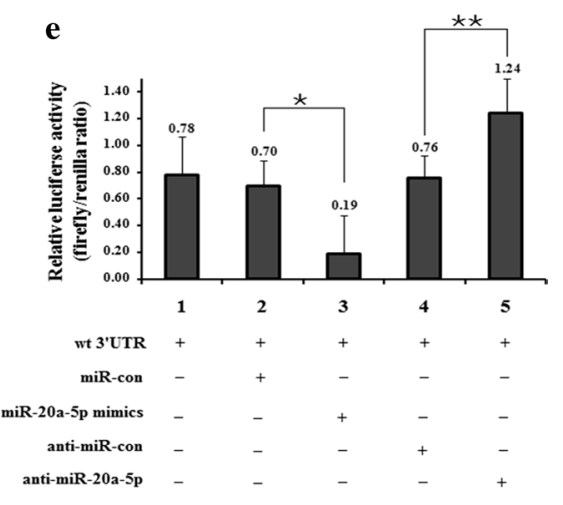

Fig. 2 Rab27B is a target of miR-20a-5p in NPC cells. Level of miR-20a-5p,Rab27B mRNA and protein levels in the miR-20a-5p mimic (5PM)-transfected CNE-2 and the miR-20a-5p antagomiR (5PA)-transfected CNE-1 cells versus the negative control (NC) cells, as determined by qRT-PCR (a, b) and western blot analyses $(\mathbf{c})$. The perfectly matched region of Rab27B gene 3'-UTR with miR-20a-5p (d). The relative luciferase activity (fold) of the reporter with wild-type (WT) Rab27B-UTR were determined in the miR-20a-5p mimic or anti or Controls transfected NPC cells. The reporter without Rab27B-UTR (Vec) was used as a reference. The Renilla luciferase activity of a co-transfected control plasmid was used to control the transfection efficacy $(\mathbf{e})$. The representative results from three independent experiments shown. ${ }^{*} P$ value $<0.05$; ${ }^{*} P$ value $<0.01$

Meanwhile, the transfection of miR-20a-5p-antagomiR into CNE-1 cells drastically raised the luciferase activity of pGL3-Rab27B-UTR WT construct (Fig. 2e). Getting together, our results strongly indicate that Rab27B is indeed a target of miR-20a-5p.

The Rab27B expression negatively correlates with the miR-20a-5p's promoting effect on the NPC radio-resistance To explore the role of Rab27B in the NPC radio-resistance, we first transfected miR-20a-5p-mimic into CNE-2 cells and tested the level of miR-20a-5p. The transfection of miR-20a-5p-mimic indeed increased the level of miR-20a-5p in the CNE-2 cells. Accompanied by the increase of miR-20a-5p, the cell survival rate was increased against the radiation treatment in the CNE-2 cells (Fig. 3a). In addition, the fluorescent immunostaining against $\gamma-\mathrm{H} 2 \mathrm{AX}$ also showed increased cell viability upon the addition of miR-20a-5p-mimic into the CNE-2 cells (Additional file 2: Figure S1). Consequently, the transfection of miR-20a-5p-mimic into the CNE-2 cells desensitizes NPC cells to irradiation (Additional file 1: Table S1). Then we transfected the si-Rab27B into CNE-2 cells and tested the effect against radiation. The transfection of si-Rab27B into CNE-2 cells indeed decreased the level of Rab27B in both mRNA $(0.57: 1)$ and protein level (0.48:1), compared to the control cells (Fig. 3b, c). Consequently, the radio-resistance of CNE-2 cells was also increased with the transfection of si-Rab27B (Fig. 3d). In addition, we transfected miR-20a-5p antagomiR into CNE-1 cells to decrease the level of miR-20a-5p, which results in a lower cell survival rate against irradiation treatment in CNE-1 cells (Fig. 3e). A similar effect was also found in the fluorescent immunostaining assays against $\gamma-\mathrm{H} 2 \mathrm{AX}$ (Additional file 2: Figure S1). Moreover, we over-expressed Rab27B by 2.23 fold (Fig. 3f, g), which also results in a lower cell survival rate against irradiation treatment in CNE-1 cells (Fig. 3h). The results correlate well with the negative regulation of Rab27B in the NPC radio-resistance. Taken together, the Rab27B gene does contribute a great deal to the miR-20a-5p's promoting effect on the NPC radio-resistance.

\section{MiR-20a-5p promotes cell migration and invasion of NPC cells}

To explore whether miR-20a-5p is involved in the metastasis of NPC cells, we compared the migration and invasion capability of CNE-1 and CNE-2 cells using the wound-healing and invasion assays, respectively. We first transfected miR-20a-5p mimics or si-Rab27B into CNE-2 cells and detected the migration and invasion. Compared to the control cells, transfection of miR-20a-5p mimic or si-Rab27B significantly increased the ability of cell migration to about 2.31- and 1.33-fold, similar to that for the invasion assays (Fig. 4a, b). Then we transfected 

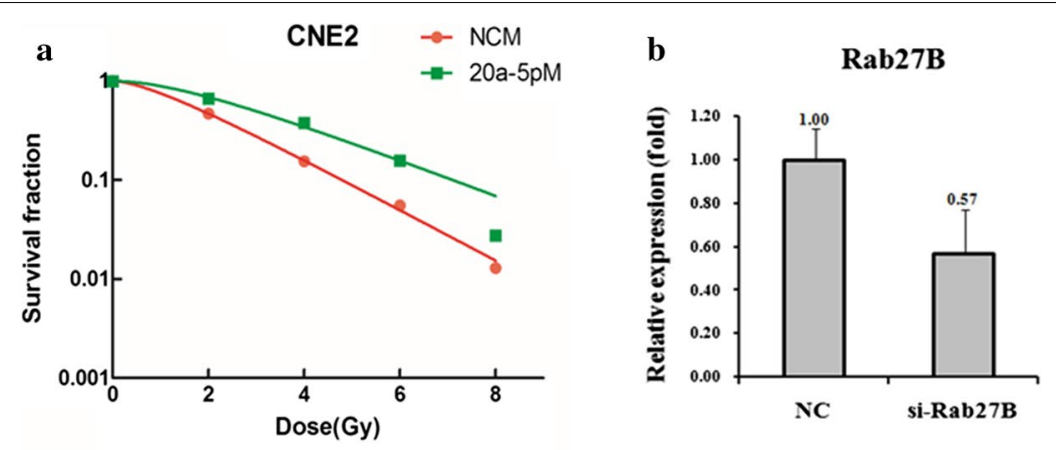

c
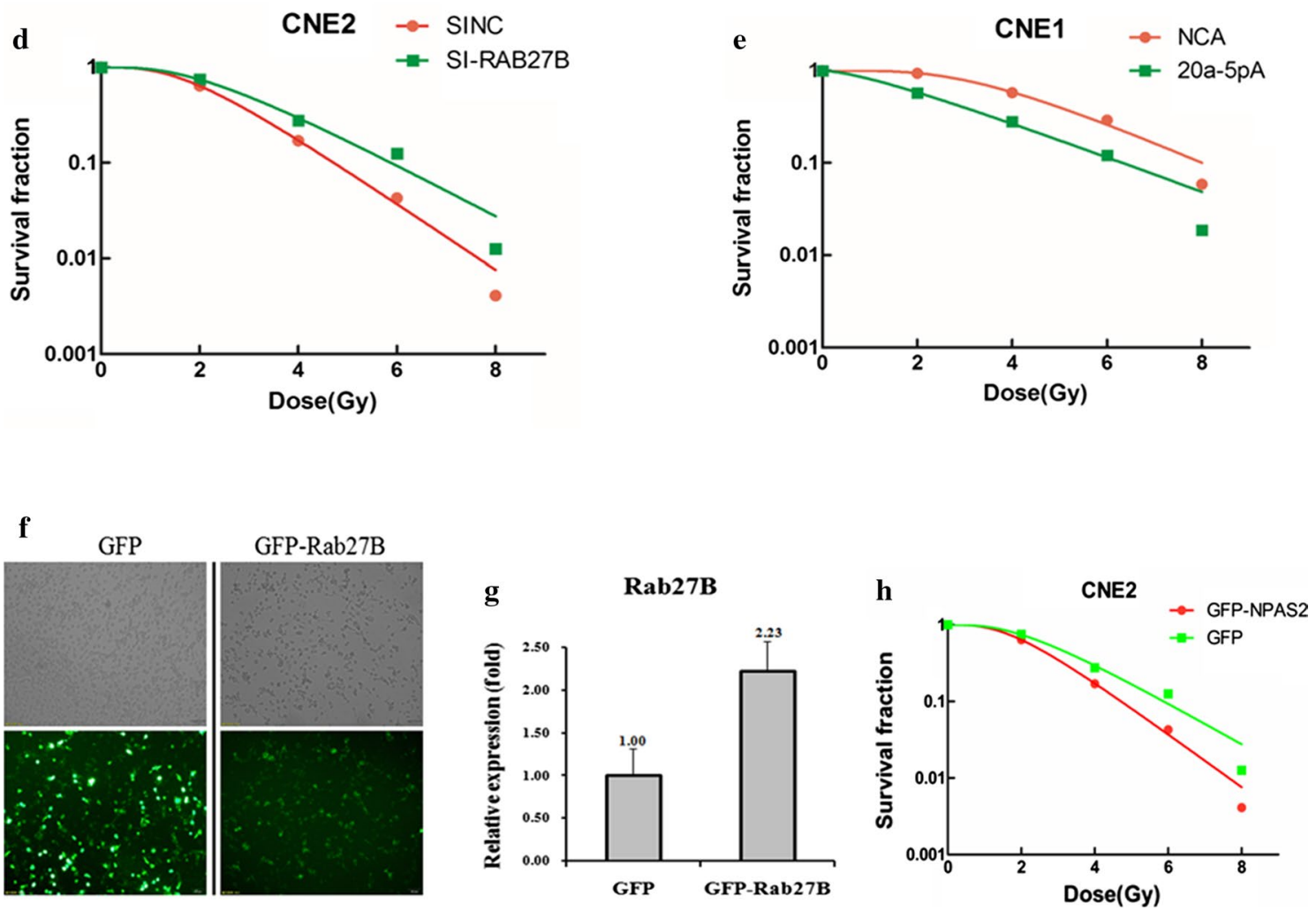

Fig. 3 Effects of a forced reversal of the miR-20a-5p or Rab27B levels on the radio-resistance of CNE-1 and CNE-2 cells. MiR-20a-5p mimic (5PM)transfected CNE-2 increases NC cells resistance to radiation treatment versus the negative control (NC) cells (a). Rab27B protein level (western blot analysis) and mRNA determined by qRT-PCR in the si-Rab27B-transfected versus the NC-transfected CNE-2 cells treated with a 4-Gy dose of radiation (b, c). Si-Rab27B-transfected CNE-2 increases NC cells resistance to radiation treatment versus the negative control (NC) cells (d). MiR-20a-5p antagomiR (5PA)-transfected CNE-1 decreases NC cells resistance to radiation treatment versus the negative control (NC) cells (e). Expression of Rab27B in overexpression construct transfected CNE-1 cells. Representative areas of CNE-1 cells transfected with GFP-Rab27B ectopic expression construct were shown and GFP was used as a negative control (f). Rab27B mRNA level (qRT-PCR analysis) in the GFP-Rab27B-transfected versus the GFP-transfected CNE-1 cells treated with a 4-Gy dose of radiation (g). GFP- Rab27B-transfected CNE-1 decreases NC cells resistance to radiation treatment versus the negative control (GFP) cells (h)

miR-20a-5p antagomiRs or over-expressed GFP-Rab27B into CNE-1 cells and also performed the same experiments. The results showed that both the migration and invasion of CNE-1 cells were decreased to some extent upon the transfection (Fig. 5a, b). All these results suggest that miR-20a-5p enhances the migration and invasion of
NPC cells, which might be conducted by the regulation of the Rab27B gene.

In line with its negative effect of Rab27B on cell migration and invasion, a siRNA-mediated Rab27B repression reduced the cell apoptosis rate from 5.97 to $3.74 \%$, indicating an elevated cell survival rate upon the addition of 

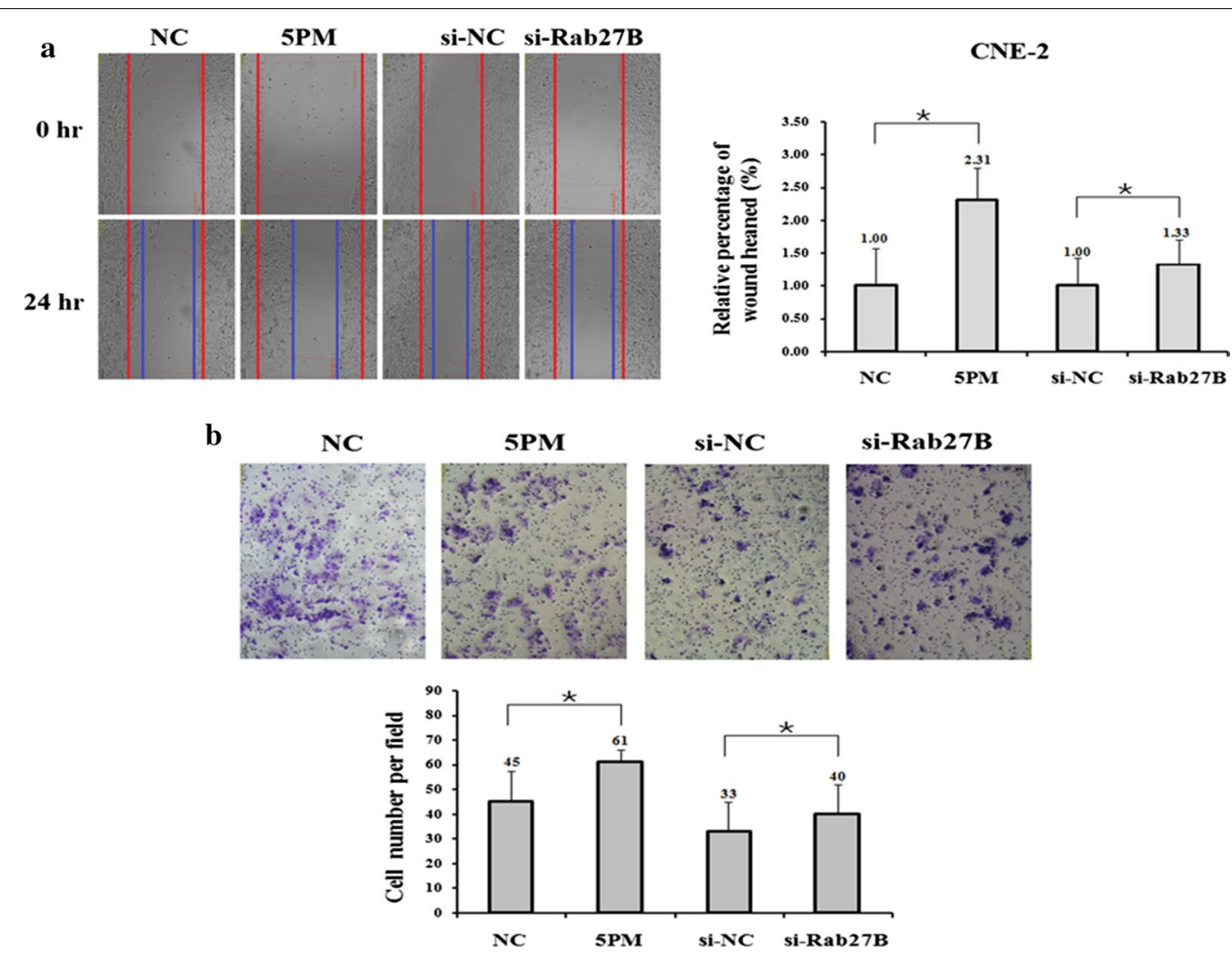

Fig. 4 Wound-healing assays was performed with transient expression of the miR-20a-5p mimic (5PM) mimic, si-Rab27B and corresponding negative control (NC) (a). Invasion assays was performed with transient expression of the miR-20a-5p mimic (5PM) mimic, si-Rab27B and corresponding negative control (NC) (b). The data are representative of three independent experiments. ${ }^{*} \mathrm{P}<0.05$

si-Rab27B into CNE-2 cells (Fig. 6). In line with the apoptosis rate, the expression of the cell apoptosis markers, PARP1 and GAPDH were significantly decreased upon the transfection of si-Rab27B into CNE-2 cells (Additional file 3: Figure S2). A similar effect was also found in the miR-20a-5p-mimic transfected CNE-2 cells (Fig. 6ac). On the other hand, the transfection of miR-20a-5pantagomiR slightly increased the cell apoptosis rate in CNE-1 cells, which also suggests a promoting effect of Rab27B on NPC radio-resistance (Fig. 6a-c). The levels of PARP1 and GAPDH were also increased, indicating a higher apoptosis rate in CNE-1 cells upon the transfection of miR-20a-5p-antagomiR (Additional file 3: Figure S2). Taken together, The Rab27B gene does contribute a great deal to the miR-20a-5p's promoting effect on the NPC radio-resistance.

\section{Discussion}

Radiation therapy is one of the major treatments against tumors as it has the advantages of being non-invasive and well tolerated. However, radiotherapy resistance is also a common occurrence that blocks the effective therapy [27]. The emerging studies have focused on the resistance mechanisms and the involved biological factors $[28,29]$. Among the factors involved, miRNAs are reported to be closely associated with tumor radiosensitivity [30-32]. The miR-20a-5p studied here is dysregulated in many human cancers [33-35], and the high level of miR-20a-5p was considered as an indicator of advanced stage, poor prognosis and chemo-therapy resistance. Of note, miR-20a was also found to induce cell radio-resistance by activating the PTEN/PI3 K/Akt signaling pathway in hepatocellular carcinoma [18]. However the knowledge of miR-20a-5p on cancer radio-resistance is still limited, especially in NPC. In this study, we found that miR-20a-5p was involved in NPC radio-resistance, probably by targetingRab27B 3'-UTR. Furthermore we demonstrated that miR-20a-5p-mediated Rab27B repression promoted the invasion and metastasis of NPC cells. Both role and mechanisms of miR-20a-5p and Rab27B in NPC radio-resistance were systematically investigated in cultured cells. Furthermore, the influence of miR-20a-5p and Rab27B on the growth of tumor xenografts was also addressed in nude mice.

Rab27B is a member of Ras-like small GTPases that modulate endocytosis and exocytosis vesicle-trafficking 


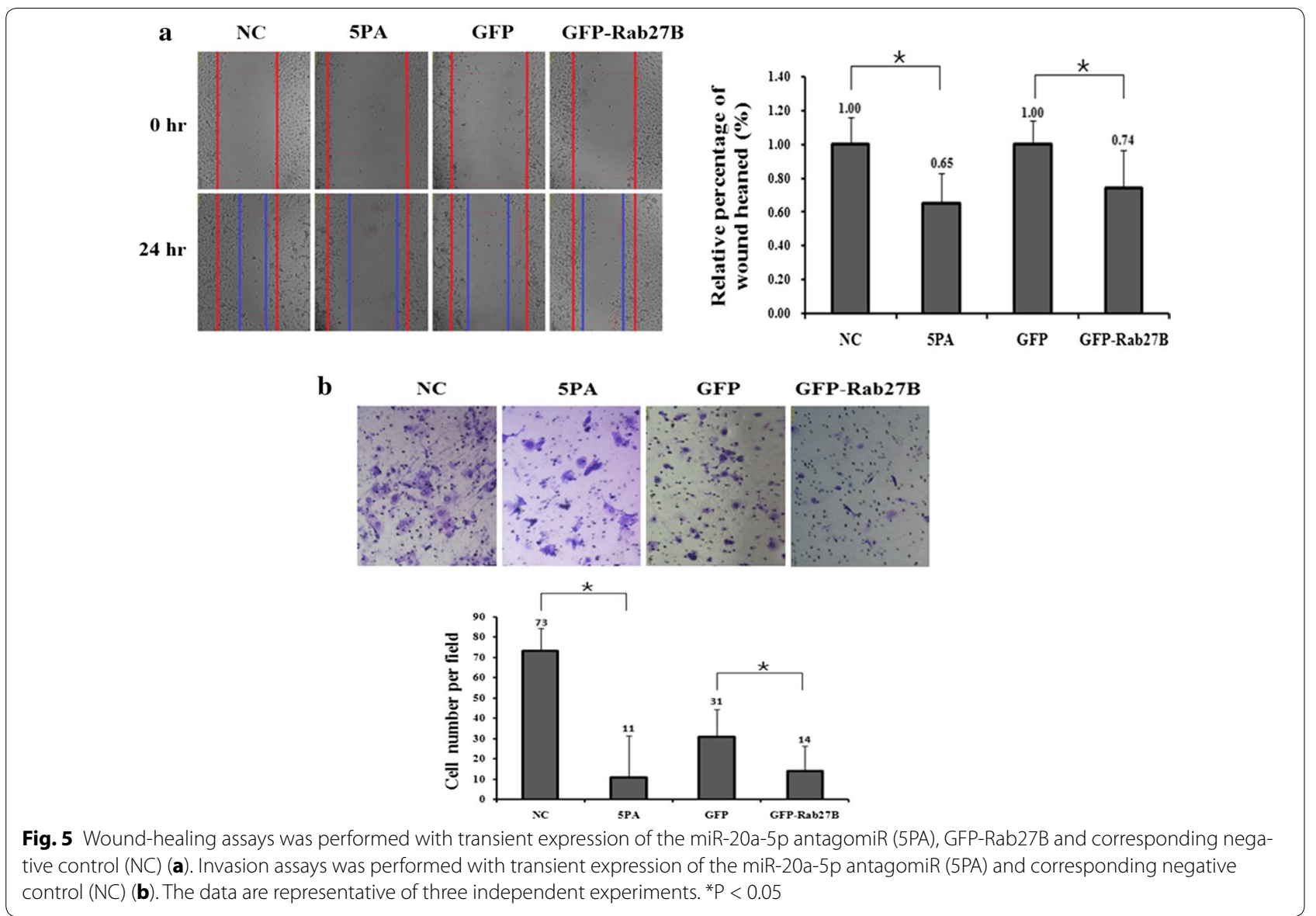

control [36-39]. Rab27B is normally expressed in a large number of secretory cells to regulate secretory pathways [40]. In addition, it is reported that aberrant expression of Rab27B is associated with several types of cancers. For examples, the increased Rab27B expression correlates with lymph node metastasis and is a marker for breast cancer progression [41, 42]. Rab27B can also be recognized as a valuable prognostic indicator for hepatocellular carcinoma patients. In addition, Rab27B regulates invasive tumor growth of colorectal cancer [43], hepatocellular carcinoma [44] and breast cancer [42, 45, 46]. Based on the above studies, Rab27B demonstrates oncogenic function and plays important roles in cancer development. However, the expression of Rab27B, as well as its role in NPC, has barely been investigated. In this study, our data suggest that Rab27B might facilitate the invasive/ 


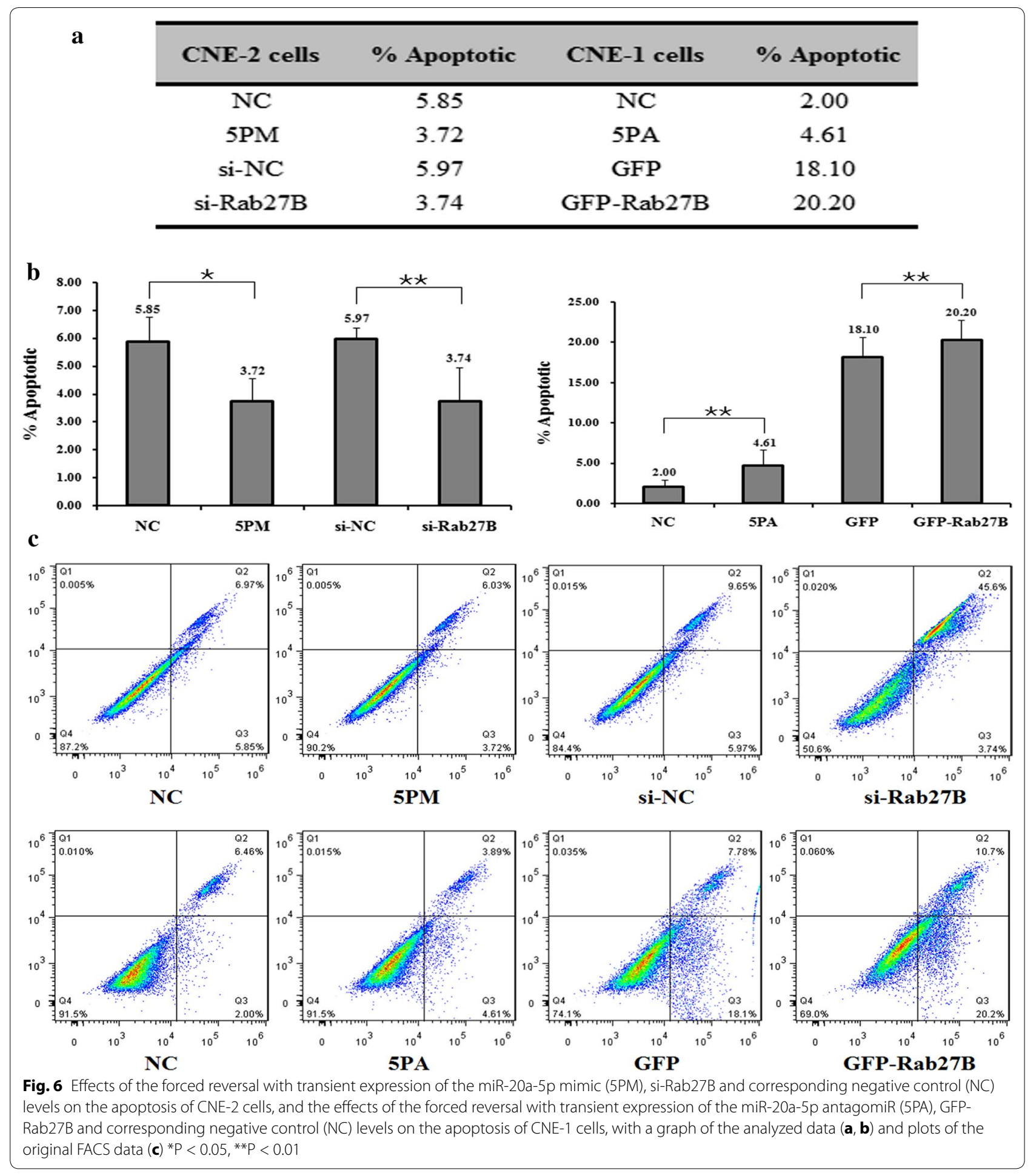

metastatic phenotypes of NPC, and thus might be treated as a novel marker for clinical diagnosis. We showed that the expression of Rab27B is associated with the radioresistance of NPC cell lines, which is mediated by miR20a-5p. Despite that the expression level of $\gamma-\mathrm{H} 2 \mathrm{AX}$ is correlated with the cell apoptosis of NPC cells, other pathways may be activated upon the exposure to the radiation, such as JNK signal pathway [47]. The detailed mechanism for the miR-20a-5p-mediated Rab27B repression of NPC radio-resistance remains to be elucidated. 


\section{Additional files}

Additional file 1: Table S1. The interested miRNA genes based on the miR-omic analysis. A dozen of miRNAs were differentially expressed in the radio-resistant and radio-sensitive cell lines based on the miR-omic analysis were showed in descending order, has-miR-20a-5p was one of them.

Additional file 2: Figure S1. MiR-20a-5p promotes NPC cell viability and sensitizes NPC cells to irradiation. $y-H 2 A X$ foci formation was determined in CNE-2 and CNE-1 cells transfected with either miR-20a-5p mimic or miR-20a-5p antagomiR $24 \mathrm{~h}$ following irradiation. DAPi staining was performed and micrographs were captured at magnification $\times 400$. The median number of foci formation is presented in bar graphs. Values are presented as the median \pm standard deviation

Additional file 3: Figure S2. The protein level of PARP and caspase3 detected by western in NCM, 5PM, NCA, 5PA, si-NC and si-Rab27B transfected CNE-2 and CNE-1 cells respectively.

\section{Abbreviations}

NPC: nasopharyngeal cancer; MiR: MicroRNA; UTR: untranslated region; Rab27B: member RAS oncogene family; 5PM: miR-20a-5p; 5PA: miR-20a-5p.

\section{Authors' contributions}

Conception and design: DBH and SH. Acquisition of data (provided animals, provided facilities, etc.): DBH, GB, YYP, XHH, YBS, YW analysis and interpretation of data (e.g., statistical analysis, biostatistics, computational analysis): GB, GDS, MC, XF. Writing, review, and/or revision of the manuscript: DBH, GB and SH. All authors read and approved the final manuscript.

\section{Author details}

${ }^{1}$ Shandong University School of Medicine, Jinan 250012, China. ${ }^{2}$ Department of Geriatrics, Anhui Provincial Hospital, Anhui Medical University, Hefei 230031, Anhui, China. ${ }^{3}$ Department of Oncology, Anhui Provincial Hospital, Anhui Medical University, Hefei 230001, China. ${ }^{4}$ Anhui Provincial Key Laboratory of Tumor Immunotherapy and Nutrition Therapy, Hefei 230001, China.

\section{Acknowledgements}

Not applicable.

\section{Competing interests}

The authors declare that they have no competing interests.

\section{Availability of data and materials}

Data sharing not applicable to this article as no datasets were generated or analysed during the current study.

\section{Consent for publication}

The authors consent for publication.

\section{Ethics statement}

Animal experiments were undertaken in accordance with the National Institutes of Health Guide for the Care and Use of Laboratory Animals. Animal research was approved by the biomedical ethics committee of Anhui Medical University, when we applying for the National Natural Science Foundation of China (No. 81471552). The animal study proposal was approved by the Institutional Animal Care and Use Committee (IACUC) of the University of Science and Technology of China. All of the mouse experimental procedures were performed in accordance with the Regulations for the Administration of Affairs Concerning Experimental Animals approved by the State Council of People's Republic of China.

\section{Funding}

This work was supported by the National Natural Science Foundation of China (No. 81471552 granted to Min Chen), Anhui Provincial Natural Science Foundation (Nos. 1408085MH156 and 1408085MH167 granted to Min Chen, Guodong Shen, respectively), and Anhui Provincial science and technology key projects (Nos. 1303063017, 1301042094 granted to Shilian Hu, Guodong
Shen, respectively). The funders had no role in the study design, data collection and analysis, decision to publish, or the preparation of the manuscript.

Received: 5 September 2016 Accepted: 28 January 2017

Published online: 01 March 2017

\section{References}

1. Liu T. Issues in the management of nasopharyngeal carcinoma. Crit Rev Oncol/Hematol. 1999;31(1):55-69.

2. Horsman MR, Bohm L, Margison GP, Milas L, Rosier JF, Safrany G, Selzer E, Verheij M, Hendry JH. Tumor radiosensitizers-current status of development of various approaches: report of an International Atomic Energy Agency meeting. Int J Radiat Oncol Biol Phys. 2006;64(2):551-61.

3. Louis C, Dewas S, Mirabel X, Lacornerie T, Adenis A, Bonodeau F, Lartigau E. Stereotactic radiotherapy of hepatocellular carcinoma: preliminary results. Technol Cancer Res Treat. 2010;9(5):479-87.

4. Seo YS, Kim MS, Yoo SY, Cho CK, Choi CW, Kim JH, Han CJ, Park SC, Lee $\mathrm{BH}, \mathrm{Kim} \mathrm{YH}$, et al. Preliminary result of stereotactic body radiotherapy as a local salvage treatment for inoperable hepatocellular carcinoma. J Surg Oncol. 2010;102(3):209-14.

5. Tse RV, Hawkins M, Lockwood G, Kim JJ, Cummings B, Knox J, Sherman M, Dawson LA. Phase I study of individualized stereotactic body radiotherapy for hepatocellular carcinoma and intrahepatic cholangiocarcinoma. J Clin Oncol. 2008;26(4):657-64.

6. Jameel JK, Rao VS, Cawkwell L, Drew PJ. Radioresistance in carcinoma of the breast. Breast. 2004;13(6):452-60.

7. Pillai RS, Bhattacharyya SN, Filipowicz W. Repression of protein synthesis by miRNAs: how many mechanisms? Trends Cell Biol. 2007;17(3):118-26.

8. Lu J, Getz G, Miska EA, Alvarez-Saavedra E, Lamb J, Peck D, Sweet-Cordero A, Ebert BL, Mak RH, Ferrando AA, et al. MicroRNA expression profiles classify human cancers. Nature. 2005;435(7043):834-8.

9. Volinia S, Calin GA, Liu CG, Ambs S, Cimmino A, Petrocca F, Visone R, lorio M, Roldo C, Ferracin M, et al. A microRNA expression signature of human solid tumors defines cancer gene targets. Proc Natl Acad Sci USA. 2006;103(7):2257-61.

10. Niemoeller OM, Niyazi M, Corradini S, Zehentmayr F, Li M, Lauber K, Belka C. MicroRNA expression profiles in human cancer cells after ionizing radiation. Radiat Oncol. 2011;6:29.

11. Weidhaas JB, Babar I, Nallur SM, Trang P, Roush S, Boehm M, Gillespie E, Slack FJ. MicroRNAs as potential agents to alter resistance to cytotoxic anticancer therapy. Cancer Res. 2007;67(23):11111-6.

12. Liao H, Xiao Y, Hu Y, Xiao Y, Yin Z, Liu L. microRNA-32 induces radioresistance by targeting DAB2IP and regulating autophagy in prostate cancer cells. Oncol Lett. 2015;10(4):2055-62.

13. Xia H, Chen S, Chen K, Huang H, Ma H. MiR-96 promotes proliferation and chemo- or radioresistance by down-regulating RECK in esophageal cancer. Biomed Pharmacother. 2014;68(8):951-8.

14. Hayashita Y, Osada H, Tatematsu Y, Yamada H, Yanagisawa K, Tomida S, Yatabe Y, Kawahara K, Sekido Y, Takahashi T. A polycistronic microRNA cluster, miR-17-92, is overexpressed in human lung cancers and enhances cell proliferation. Cancer Res. 2005;65(21):9628-32.

15. Kutay H, Bai S, Datta J, Motiwala T, Pogribny I, Frankel W, Jacob ST, Ghosha K. Downregulation of miR-122 in the rodent and human hepatocellular carcinomas. J Cell Biochem. 2006;99(3):671-8.

16. Li X, Zhang Z, Yu M, Li L, Du G, Xiao W, Yang H. Involvement of miR-20a in promoting gastric cancer progression by targeting early growth response 2 (EGR2). Int J Mol Sci. 2013:14(8):16226-39.

17. Wagner-Ecker M, Schwager C, Wirkner U, Abdollahi A, Huber PE. MicroRNA expression after ionizing radiation in human endothelial cells. Radiat Oncol. 2010;5:25.

18. Zhang Y, Zheng L, Ding Y, Li Q, Wang R, Liu T, Sun Q, Yang H, Peng S, Wang W, et al. MiR-20a induces cell radioresistance by activating the PTEN/ PI3 K/Akt signaling pathway in hepatocellular carcinoma. Int J Radiat Oncol Biol Phys. 2015;92(5):1132-40.

19. Li J, Tu Z, Shen Z, Xia Y, He Y, Liu S, Chen C. Quantitative measurement of optical attenuation coefficients of cell lines CNE1, CNE2, and NP69 using optical coherence tomography. Lasers Med Sci. 2013;28(2):621-5. 
20. Tarazona S, Garcia-Alcalde F, Dopazo J, Ferrer A, Conesa A. Differential expression in RNA-seq: a matter of depth. Genome Res. 2011;21(12):2213-23

21. Pu Y, Zhao F, Wang H, Cai W, Gao J, Li Y, Cai S: MiR-34a-5p promotes the multi-drug resistance of osteosarcoma by targeting the CD117 gene. Oncotarget 2016.

22. Liu K, Huang J, Xie M, Yu Y, Zhu S, Kang R, Cao L, Tang D, Duan X. MIR34A regulates autophagy and apoptosis by targeting HMGB1 in the retinoblastoma cell. Autophagy. 2014;10(3):442-52.

23. Lv L, Deng H, Li Y, Zhang C, Liu X, Liu Q, Zhang D, Wang L, Pu Y, Zhang H, et al. The DNA methylation-regulated miR-193a-3p dictates the multichemoresistance of bladder cancer via repression of SRSF2/PLAU/HIC2 expression. Cell Death Dis. 2014;5:e1402.

24. He BF, Sun AM, Huang BY, Wang WJ, Zheng XK, Luo RC. Gefitinib enhances the radiosensitivity of nasopharyngeal carcinoma cell line CNE2 in vitro. Nan fang yi ke da xue xue bao. J South Med Univ. 2011;31(6):991-4.

25. Hui ZG, Li YX, Yang WZ, Wu JX, Yu ZH. Abrogation of radiation-induced $\mathrm{G} 2$ arrest and radiosensitization by 7-hydroxystaurosporine (UCN-01) in human nasopharyngeal carcinoma cell line. Ai zheng Aizheng. Chin J Cancer. 2003;22(1):6-10.

26. Long $Z$, Wang $B$, Tao $D$, Huang $Y$, Tao Z. Hypofractionated radiotherapy induces miR-34a expression and enhances apoptosis in human nasopharyngeal carcinoma cells. Int J Mol Med. 2014;34(5):1388-94.

27. Paller CJ, Antonarakis ES, Eisenberger MA, Carducci MA. Management of patients with biochemical recurrence after local therapy for prostate cancer. Hematol/Oncol Clin North Am. 2013;27(6):1205-19.

28. Orth M, Lauber K, Niyazi M, Friedl AA, Li M, Maihofer C, Schuttrumpf L, Ernst A, Niemoller OM, Belka C. Current concepts in clinical radiation oncology. Radiat Environ Biophys. 2014;53(1):1-29.

29. Kaliberov SA, Buchsbaum DJ. Chapter seven-Cancer treatment with gene therapy and radiation therapy. Adv Cancer Res. 2012;115:221-63.

30. Oh JS, Kim JJ, Byun JY, Kim IA. Lin28-let7 modulates radiosensitivity of human cancer cells with activation of K-Ras. Int J Radiat Oncol Biol Phys. 2010;76(1):5-8.

31. Wang P, Zhang J, Zhang L, Zhu Z, Fan J, Chen L, Zhuang L, Luo J, Chen $\mathrm{H}$, Liu L, et al. MicroRNA 23b regulates autophagy associated with radioresistance of pancreatic cancer cells. Gastroenterology. 2013;145(5):1133-43.

32. Zhang Y, Zheng L, Huang J, Gao F, Lin X, He L, Li D, Li Z, Ding Y, Chen L. MiR-124 Radiosensitizes human colorectal cancer cells by targeting PRRX1. PLOS ONE. 2014;9(4):e93917.

33. Cheng D, Zhao S, Tang H, Zhang D, Sun H, Yu F, Jiang W, Yue B, Wang J, Zhang M, Yu Y. MicroRNA-20a-5p promotes colorectal cancer invasion and metastasis by downregulating Smad4. Oncotarget. 2016;7(29):45199-213. doi:10.18632/oncotarget.9900.
34. Calvano Filho CM, Calvano-Mendes DC, Carvalho KC, Maciel GA, Ricci MD, Torres AP, Filassi JR, Baracat EC. Triple-negative and luminal A breast tumors: differential expression of miR-18a-5p, miR-17-5p, and miR20a-5p. Tumour Biol. 2014;35(8):7733-41.

35. Xu Q, Dong QG, Sun LP, He CY, Yuan Y. Expression of serum miR-20a-5p, let-7a, and miR-320a and their correlations with pepsinogen in atrophic gastritis and gastric cancer: a case-control study. BMC clinical pathology. 2013;13:11.

36. Fukuda M. Regulation of secretory vesicle traffic by Rab small GTPases. Cell Mol Life Sci. 2008;65(18):2801-13.

37. Pereira-Leal JB, Seabra MC. Evolution of the Rab family of small GTPbinding proteins. J Mol Biol. 2001;313(4):889-901.

38. Zerial M, McBride H. Rab proteins as membrane organizers. Nature Rev Mol Cell Biol. 2001;2(2):107-17.

39. Pfeffer SR. Structural clues to Rab GTPase functional diversity. J Biol Chem. 2005:280(16):15485-8.

40. Johnson JL, Brzezinska AA, Tolmachova T, Munafo DB, Ellis BA, Seabra MC, Hong H, Catz SD. Rab27a and Rab27b regulate neutrophil azurophilic granule exocytosis and NADPH oxidase activity by independent mechanisms. Traffic. 2010;11(4):533-47.

41. Hendrix A, Braems G, Bracke M, Seabra M, Gahl W, De Wever O, Westbroek W. The secretory small GTPase Rab27B as a marker for breast cancer progression. Oncotarget. 2010;1(4):304-8.

42. Zhang JX, Huang XX, Cai MB, Tong ZT, Chen JW, Qian D, Liao YJ, Deng HX, Liao DZ, Huang MY, et al. Overexpression of the secretory small GTPase Rab27B in human breast cancer correlates closely with lymph node metastasis and predicts poor prognosis. J Transl Med. 2012;10:242.

43. Bao J, Ni Y, Qin H, Xu L, Ge Z, Zhan F, Zhu H, Zhao J, Zhou X, Tang X, et al. Rab27b is a potential predictor for metastasis and prognosis in colorectal cancer. Gastroenterol Res Pract. 2014;2014:913106.

44. Dong WW, Mou Q, Chen J, Cui JT, Li WM, Xiao WH. Differential expression of Rab27A/B correlates with clinical outcome in hepatocellular carcinoma. World J Gastroenterol. 2012;18(15):1806-13.

45. Hendrix A, Maynard D, Pauwels P, Braems G, Denys H, Van den Broecke R, Lambert J, Van Belle S, Cocquyt V, Gespach C, et al. Effect of the secretory small GTPase Rab27B on breast cancer growth, invasion, and metastasis. J Natl Cancer Inst. 2010;102(12):866-80.

46. Hendrix A, Sormunen R, Westbroek W, Lambein K, Denys H, Sys G, Braems G, Van den Broecke R, Cocquyt V, Gespach C, et al. Vacuolar $\mathrm{H}+$ ATPase expression and activity is required for Rab27B-dependent invasive growth and metastasis of breast cancer. Int J Cancer J Int Cancer. 2013;133(4):843-54

47. Picco V, Pages G. Linking JNK activity to the DNA damage response. Genes Cancer. 2013:4(9-10):360-8.

\section{Submit your next manuscript to BioMed Central and we will help you at every step:}

- We accept pre-submission inquiries

- Our selector tool helps you to find the most relevant journal

- We provide round the clock customer support

- Convenient online submission

- Thorough peer review

- Inclusion in PubMed and all major indexing services

- Maximum visibility for your research

Submit your manuscript at www.biomedcentral.com/submit 